\title{
Fasting, Diabetes, and Optimizing Health Outcomes for Ramadan Observers: A Literature Review
}

Hadi A. Almansour · Betty Chaar · Bandana Saini

Received: November 23, 2016 / Published online: February 8, 2017

(C) The Author(s) 2017. This article is published with open access at Springerlink.com

\section{ABSTRACT}

Introduction: Globally, and in Australia, diabetes has become a common chronic health condition. Diabetes is also quite prevalent in culturally and linguistically diverse pockets of the Australian population, including Muslims. There are over 90 million Muslims with diabetes worldwide. Diabetes management and medication use can be affected by religious practices such as fasting during Ramadan. During Ramadan, Muslims refrain from oral or intravenous substances from sunrise to sunset. This may lead to many potential health or medication-related risks for patients with diabetes who observe this religious practice. This literature review aimed to explore (1) health care-related interventions and (2) intentions, perspectives, or needs of health care professionals (HCPs) to provide clinical services to patients with diabetes while fasting during Ramadan with a view to improve health outcomes for those patients.

Methods: Using a scoping review approach, a comprehensive search was conducted. Databases searched systematically included PubMed,

Enhanced content To view enhanced content for this article go to http://www.medengine.com/Redeem/ 4887F06060CC1453.

H. A. Almansour $(\bowtie) \cdot$ B. Chaar · B. Saini

Faculty of Pharmacy, University of Sydney, Sydney, Australia

e-mail: halm7156@uni.sydney.edu.au
Medline, Embase, and International Pharmaceutical Abstracts. Studies published in English that described interventions or intentions to provide interventions regarding diabetes and Ramadan fasting were included.

Results: Fourteen published articles that met the inclusion criteria were retrieved and content analyzed. Of those, nine intervention studies regarded diabetes management education. Five studies described professional service intention, four of which were related to the role of pharmacists in diabetes management in Qatar, Australia, and Egypt, and one French study examined the general practitioners' (GPs) experiences in diabetes management for Ramadan observers. The intervention studies had promising outcomes for diabetes management during Ramadan. Effect sizes for improvement in $\mathrm{HbA1c}$ post intervention ranged widely from -1.14 to 1.7 . Pharmacists appeared to be willing to participate in programs to help fasting patients with diabetes achieve a safe therapeutic outcome. Service intention studies highlighted pharmacists' and GPs' need for training prior to providing services from a clinical as well as cultural competence perspective.

Conclusion: Interventions research in this area requires robustly designed and structured interventions that can be tested in different contexts. This literature review revealed many gaps regarding diabetes management in Ramadan. Health professionals are willing to provide services for fasting diabetes patients, but need upskilling. 
Keywords: Fasting; HbA1c; Hypoglycemia; Pharmacist; Ramadan; Type 1 diabetes; Type 2 diabetes

\section{INTRODUCTION}

Internationally, the prevalence of diabetes is approximately $8.3 \%$; 382 million people live with diabetes [1]. Of these, nearly 90 million are Muslims [2]. Diabetes is also increasingly becoming a common disease among the Australian adult population. In 2011-12, for example, $4.7 \%$ of the Australian population were reported to have type 2 diabetes (T2D) [3]. Diabetes is particularly prevalent in culturally and linguistically diverse (CALD) Australians. Many of these CALD populations follow different religious beliefs. Islam is one of the key religions followed by CALD populations. For example many of those who have migrated to Australia from the Indian subcontinent, central and far east Asian countries (Indonesia/Malaysia), Europe, and from the middle-eastern and North African regions are Muslims [4]. There has been an estimated $69 \%$ increase in the Australian Muslim population from 2001 to 2011, with the total current number of Muslims believed to be approximately 476,300 [5]. Although the prevalence of diabetes in Australian Muslims is not known directly, indirect markers point to the fact that diabetes may be fairly common in this population. Studies indicating, for example, that Australian women and men born in the Middle East or North Africa were 2.4 and 3.6 times more likely to have diabetes than those who were born in Australia [6]. Given that about $42 \%$ of Australian Muslims are of North African or Middle Eastern origin, these data suggest the possibility that diabetes may be quite prevalent in Australian Muslims [4]. This population group is also likely to have socio-religious practices that may impact on their beliefs about health and health management practices [6]. Religious fasts, such as Ramadan, are a key example of this issue.

Fasts may influence the body's homeostatic rhythms and in those taking chronic medications, fasts may upset established pharmacokinetic and pharmacodynamics disposition patterns of medications [5-7]. Fasting has the potential to affect glucose control of patients who have diabetes mellitus and it can disrupt the actions of antidiabetic medications [7]. Ramadan is a well-known fast observed by Muslims. Ramadan occurs in the ninth month of the lunar calendar in Islam and lasts 29-30 days $[8,9]$. Fasting in Ramadan is one of the principal pillars of Islam; it requires Muslims to fast during daylight hours, abstaining from all food and drink, as well as substances, such as medicines, taken orally or intravenously $[10,11]$. Fasting people usually have a post-fast meal (Iftar) after sunset and a pre-fast meal before sunrise (spelt varying in English as Sohour, Suhoor, Suhur, or Sehri) [8, 10]. The absolute refraining from food and drink between sunrise and sunset can lead to disruption of homoeostasis [10]. It is worthwhile to consider the impact of fasting on the health of Ramadan observers, as these observers have been reported to have higher prevalence of diabetes. There are, for example, more than 50 million Muslims worldwide who observe Ramadan although they live with diabetes [12]. For instance, an epidemiological study mapping diabetes patients observing the Ramadan fast $(n=12,914)$ in 13 Muslim countries indicated that almost $79 \%$ of patients with T2D observed the fast of Ramadan [12].

Diabetes is actually the most common illness studied with regard to Ramadan [13-18]. Fasting has the obvious potential to disrupt diabetes management as a result of the reduced food, drink, and medication intake during certain hours [17]. The practice of observing the month-long fast can affect glucose control [19]. This could lead to avoidable health problems. One of the potential adverse effect risks of taking antidiabetic medications and fasting can be hypoglycemia. Other issues that may occur include hyperglycemia, hyperglycemic-hyperosmolar state, and diabetic ketoacidosis $[13,20]$. Several studies have attempted to document the effect of fasting on diabetes. A prospective clinical trial was conducted in Iran to examine the impact of Ramadan fasting on glycemic control in T2D patients $(n=88)$. The trial reported that glycemic control deteriorated 
significantly among T2D patients who opted to fast during Ramadan. Nonetheless, hemoglobin A1c (HbA1c) reduced significantly a month after Ramadan from $9.4 \pm 2 \%$ to $8.4 \pm 2.5 \%$ $(p<0.001)$ [21]. On the other hand, a recent review highlighted that in insulin-dependent patients with diabetes, severe hyperglycemia and ketoacidosis were insignificant concerns, and only minor hypoglycemic episodes were evident in this population, when they reported observing Ramadan [22].

Several studies have illustrated the importance and positive effects of regular glucose monitoring, dietary counselling, drug dosage and timing alteration, and patient education during Ramadan [23, 24]. The International Group for Diabetes and Ramadan (IGDR) consensus diabetes management recommendations during Ramadan (2015 update) reported by Ibrahim et al. suggest several recommendations for adverse event prevention strategies during Ramadan as shown in Table 1 [24]. Also guidelines for medication management during Ramadan have been suggested by a few groups, and key points are summarized in Table 2 .

Ahmedani et al. demonstrated in a multicenter prospective study $(n=682$ patients with diabetes) that most participating patients fasted without any serious acute adverse events during Ramadan when the recommendations mentioned above were provided [25]. In this study, for example, $91 \%$ of fasting patients had their drug dose/timing altered, and physical activity patterns were downgraded from moderate/ heavy to lighter levels of exercise; changes were overseen by medical practitioners [25].

One of the health professionals that could play a key role in optimizing the health of those with T2D observing Ramadan may be community pharmacists. Pharmacists are usually consulted far more often than physicians. They are also usually available for consultation without appointments, and in most cases health consults are provided without a fee for service. Robust data from multisite trials suggest that pharmacists' intervention for patients with diabetes can improve clinical and humanistic outcomes for patients [26-31]. However, there has not been much research conducted in Australia on fasting patients with diabetes, nor are there specific medical/allied health professional guidelines for the management of T2D in patients observing Ramadan in Australia. The first exploratory study into pharmacists' perspectives about their role in care of patients with diabetes observing Ramadan was conducted in 2015 [32]. Findings of this qualitative work indicated that pharmacists do not proactively provide care for T2D Ramadan observers, but are keen to do so if supported by training and practice frameworks [32]. To develop guidelines and interventions to optimize

Table 1 Recommendations to prevent diabetes-related adverse events risks during Ramadan (adapted from Ibrahim et al. [24])

Recommendations to prevent adverse events risks

Blood glucose monitoring several times a day depending on treatment regimen for a month prior to Ramadan

Consultation with HCPs for changing medications based at least 1 month before Ramadan begins

Avoiding large pre-dawn (Sohour) meals

Avoiding vigorous physical activities during fasting time

Recording blood glucose readings regularly during Ramadan to determine the occurrence of hypoglycemia

Breaking the fast and eating snacks immediately if hypoglycemic symptoms appear. Further recommendations for hypoglycemia treatment involve consuming $15 \mathrm{~g}$ of carbohydrates such as half a cup of orange, apple juice or regular soda, three or four glucose tablets, a table spoon of honey or sugar, five or six hard candies, a cup of milk, or a serving of glucose gel

HCP health care professional 


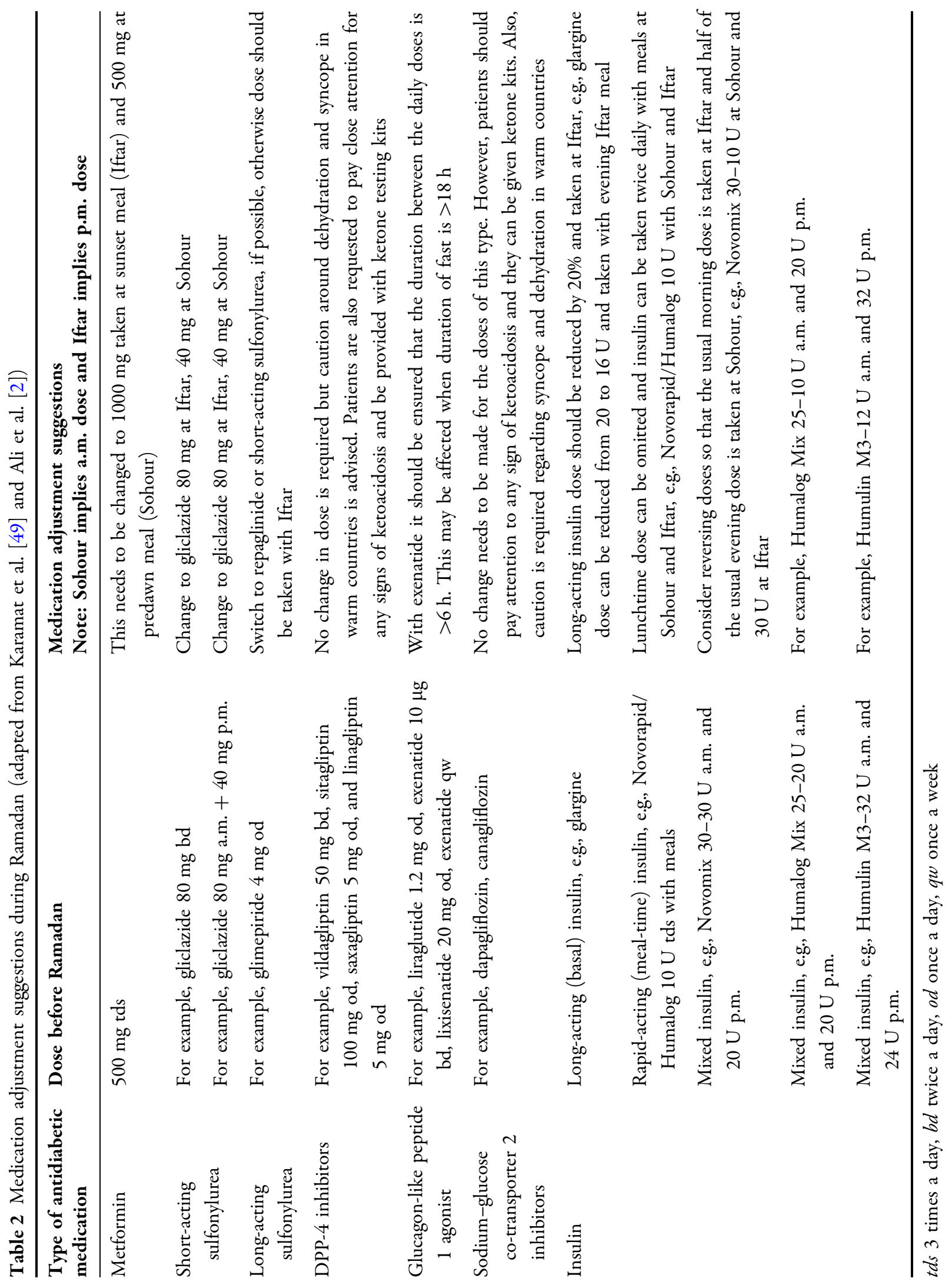


diabetes management during Ramadan in Australia, an important step would be to utilize research from international settings. International reviews have been conducted to investigate pharmaceutical interventions (e.g., medicine related trials) for patients observing Ramadan, but none have looked at educational or supportive interventions provided by health professionals [1]. This literature review aimed to explore (1) health care-related interventions and (2) intentions, perspectives, or needs of health care professionals (HCPs) to provide clinical services to patients with diabetes while fasting during Ramadan with a view to improving health outcomes for those patients.

\section{METHODS}

\section{Description of Search Strategy for Literature Review}

Given the diverse nature of interventions expected, a scoping review method was applied for the conduct of this review. A scoping review generally maps out the literature to address a broader research question and clarifies the key concepts of a research area. It helps identify gaps in the research topic based on the available literature [33]. Studies exploring the effects of fasting on patients with diabetes were searched in various online libraries and databases such as PubMed, Medline, CINAHL, EMBASE International Pharmaceutical Abstracts, and COCHRANE. The search initially used MeSH terms and keywords in combination, such as "[fasting OR Ramadan], [diabetic patients OR diabetes], [fasting in Australia OR Ramadan in Australia], [diabetes AND Ramadan], [diabetes AND Fasting], [diabetic patients AND Ramadan], [diabetic patients AND Fasting], [diabetic patients AND Fasting AND health care professionals (HCPs)]," which yielded 1469 articles. Filtration was then applied by using keywords and Boolean operands, e.g., "Fasting during Ramadan AND Diabetic patients," which resulted in 592 articles. The search was further refined by using key terms in combination "Fasting AND diabetic patients." The reference lists of articles at this stage were scoured for further relevant articles. Duplicate removal was followed by application of inclusion/exclusion criteria to all identified abstracts by the research team. Selected articles were read by the first and last author and data extracted using a tabular framework.

\section{Exclusion and Inclusion Criteria}

\section{Inclusion Criteria}

Only articles published in the English language from 1986 to August 2016 were selected for the review. The period spanned 30 years as there appeared to be limited research published on the topic, so a broader period was selected to encompass as much research as possible in the scope of our review. The articles selected were reviewed to ensure that they were original studies, and that they were published in peer-reviewed journals. Interventions delivered by all health professionals were included in the review, with a specific focus on those delivered by pharmacists. Intervention research is informed by needs analysis conducted pre-intervention. A key component of needs analyses includes the willingness of providers to engage in intervention delivery. Therefore, research conforming to this description was also included in the review, in addition to intervention trials.

\section{Exclusion Criteria}

Several exclusion criteria were applied. For example, research pertaining to other health conditions or fasts other than Ramadan was excluded from selection. Literature reviews, consensus guidelines, and case or meeting reports were also excluded.

\section{RESULTS}

A total of 596 articles were extracted from the original search. Upon further refining, as shown in Fig. 1, 14 relevant articles were obtained and included within this literature review. The study author, year, country, research methods, sample size, response rate, and the outcomes of the study were tabulated (Tables 3,4 ). These 14 
studies had utilized heterogeneous research methods including cross-sectional observational surveys or qualitative data gathering. In most studies data were collected via self-administered survey instruments, focus groups sessions, and face-to-face or telephone interviews.

Of the 14 articles, nine involved intervention studies regarding diabetes management education, and a further four studies were related to the role of pharmacists in providing or being willing to provide diabetes management interventions in Qatar, Australia, and Egypt. A French study that examined the GPs' experiences in diabetes management for Ramadan observers was also included in the review.

\section{Intervention-Based Studies}

These studies led to safer fasting during Ramadan, weight loss, and improved glycemic control among the intervention group. The care provided in some of the interventions, however, was not standardized; and some studies did not attempt to quantify the effect of interventions.

None of these studies had a robust design and response rates were either low or not provided. Power calculations were not reported in

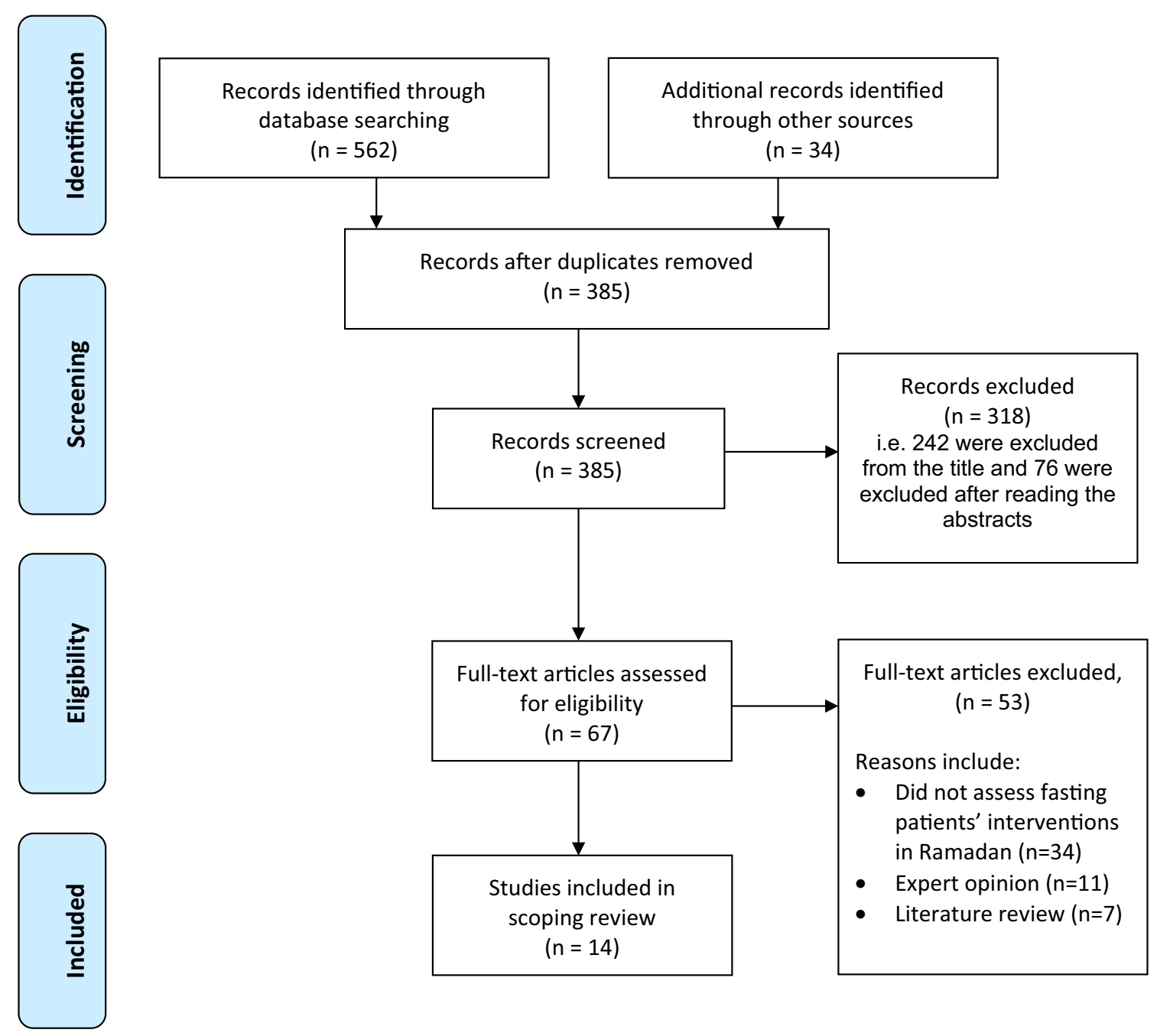

Fig. 1 Flow chart of the search strategy 


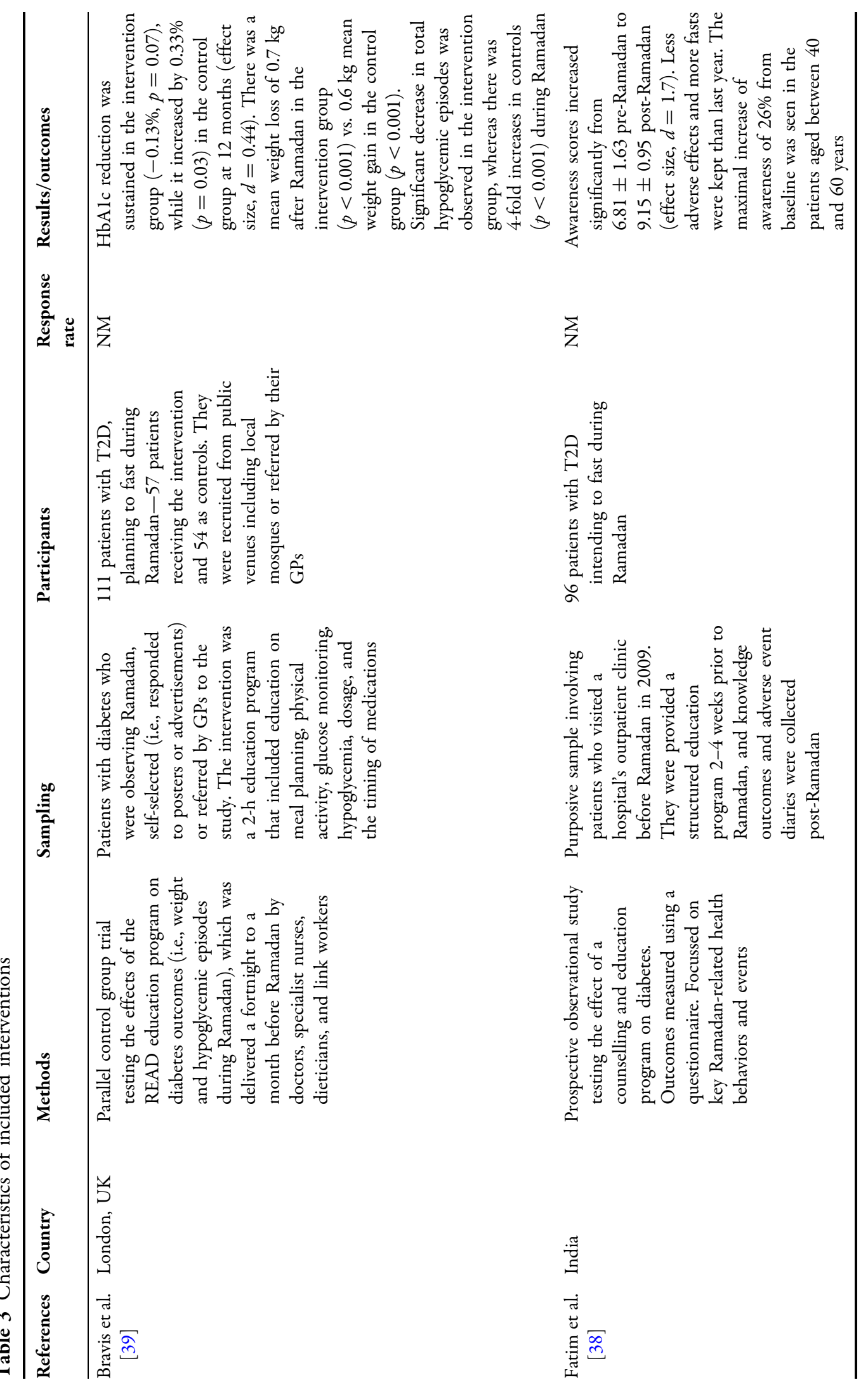




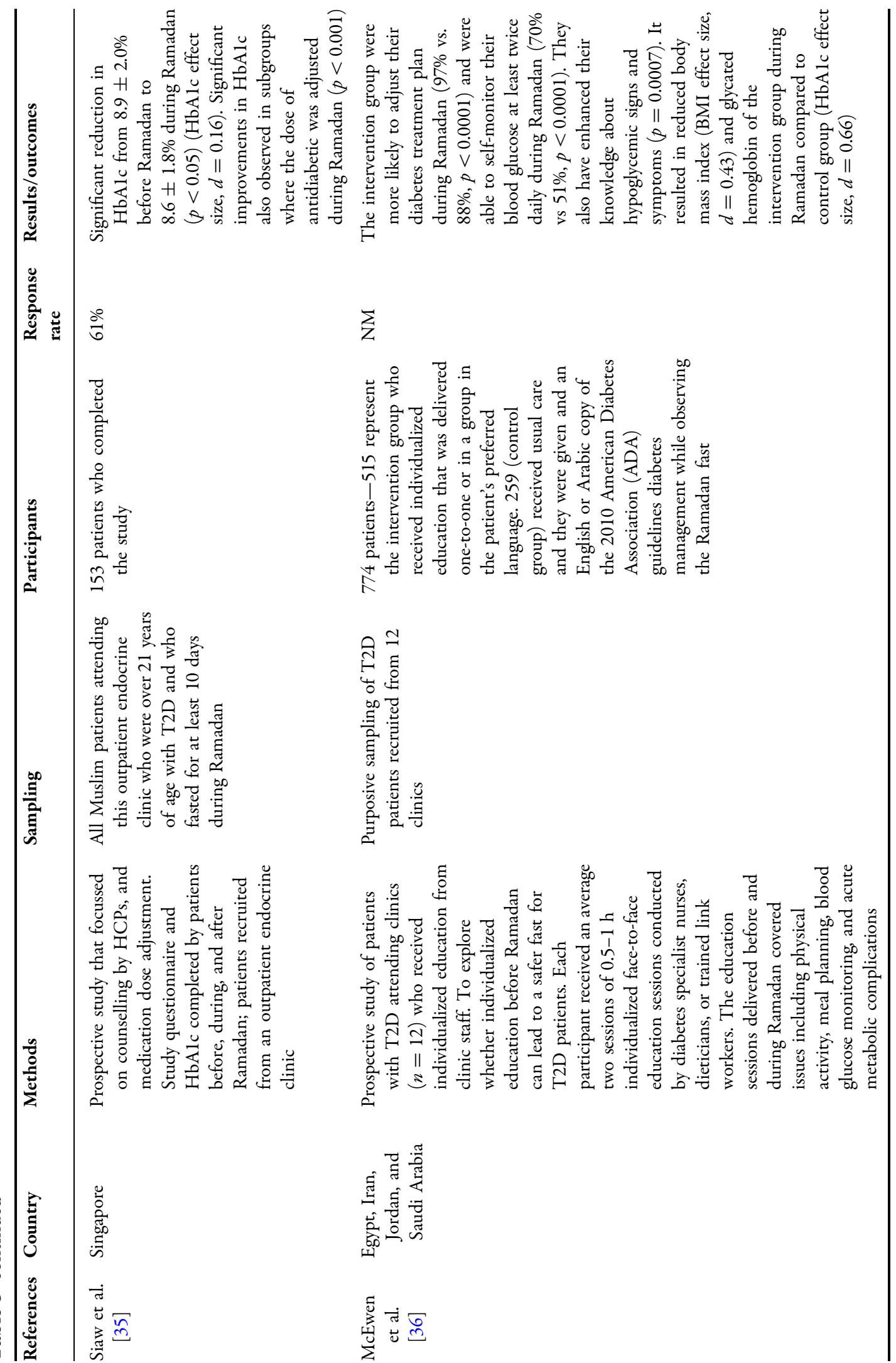




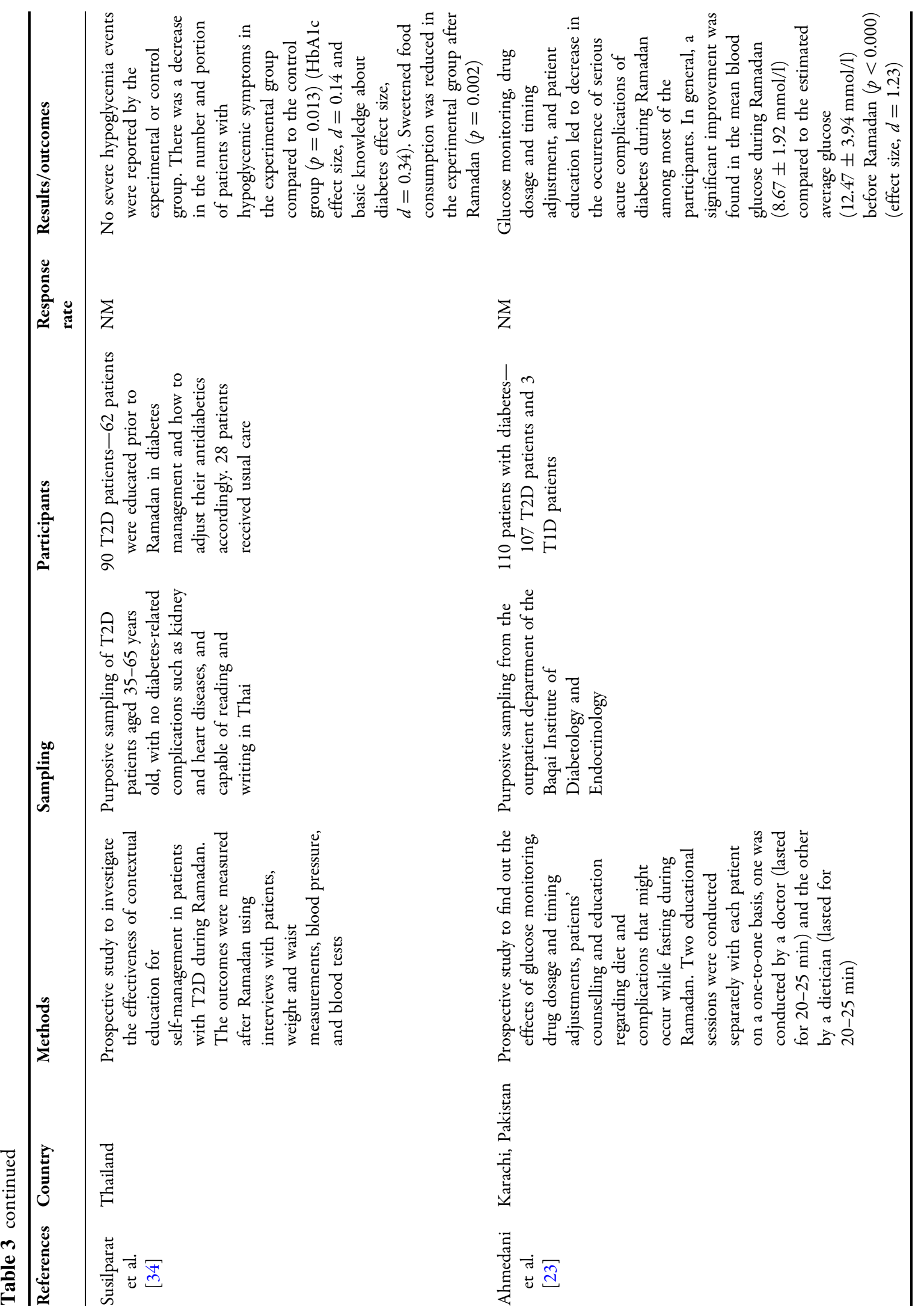




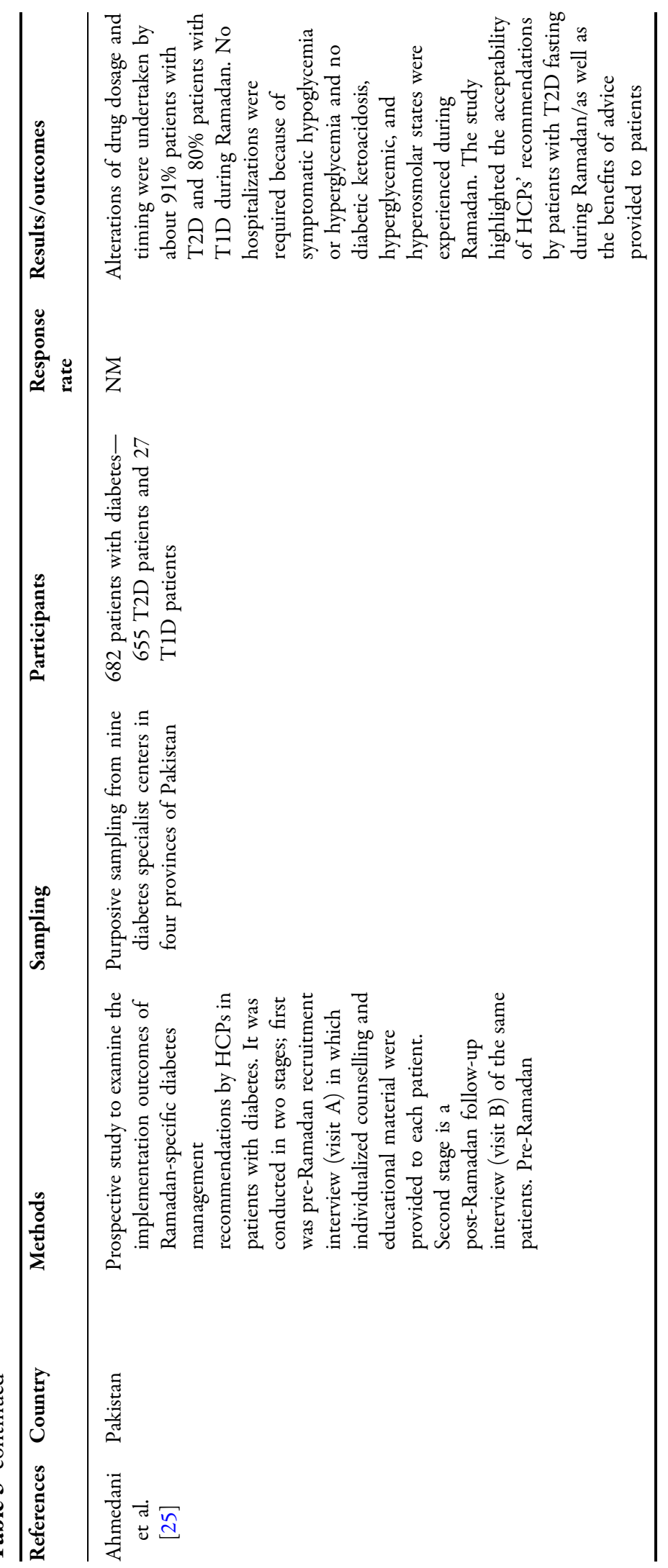




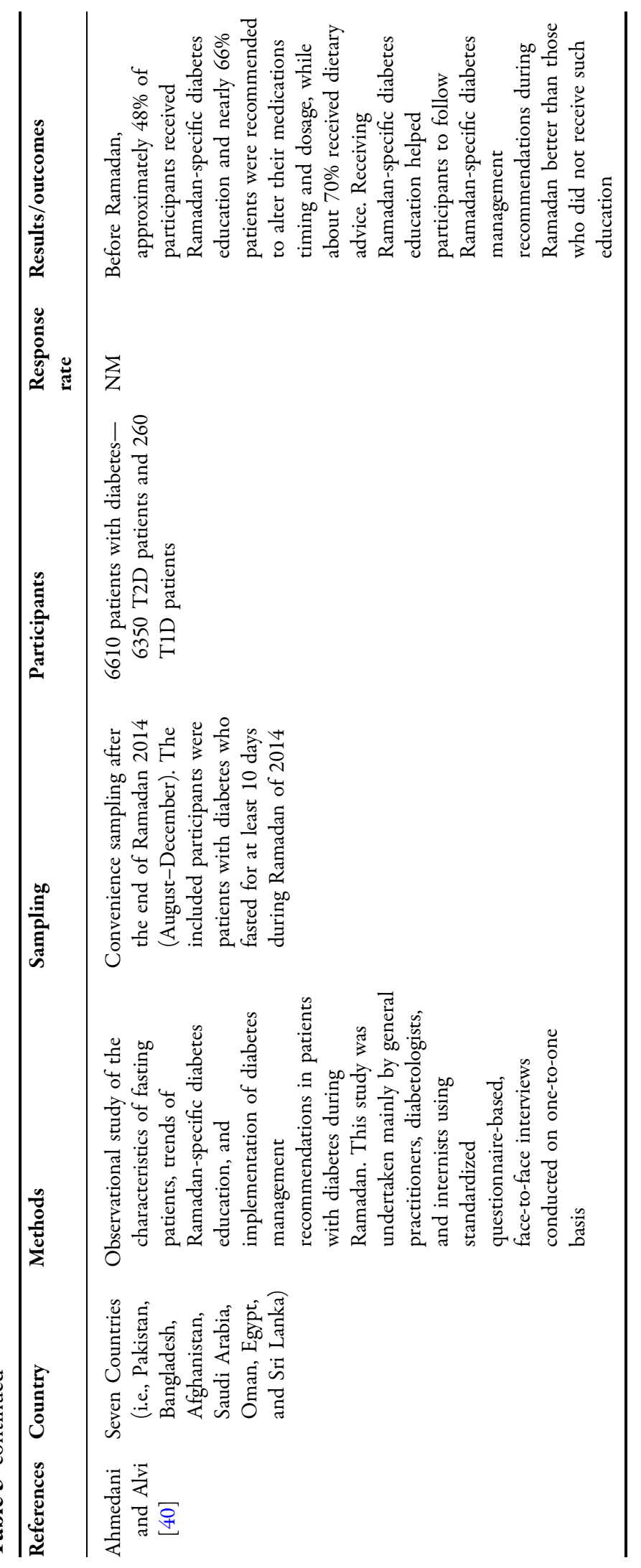




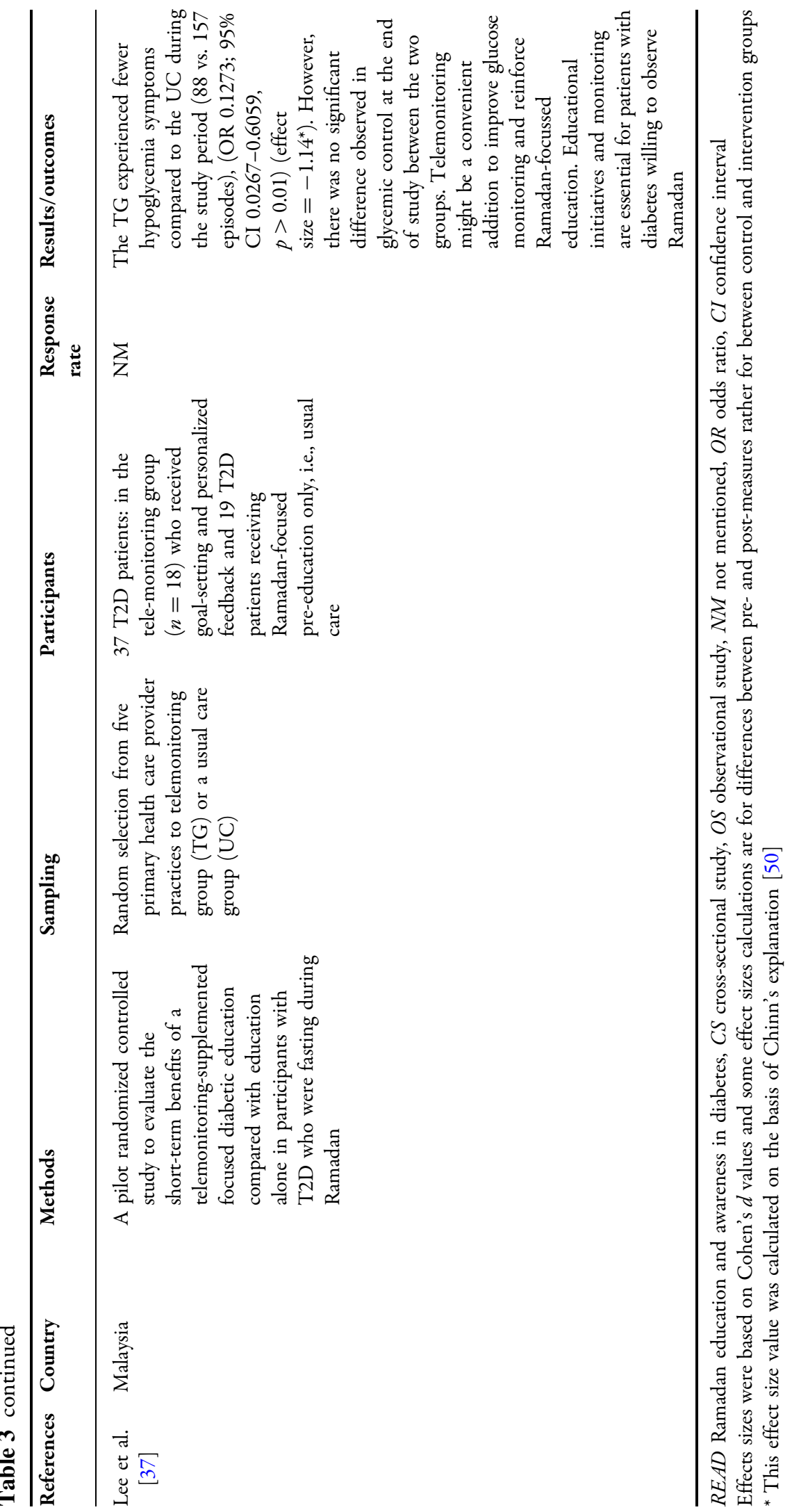




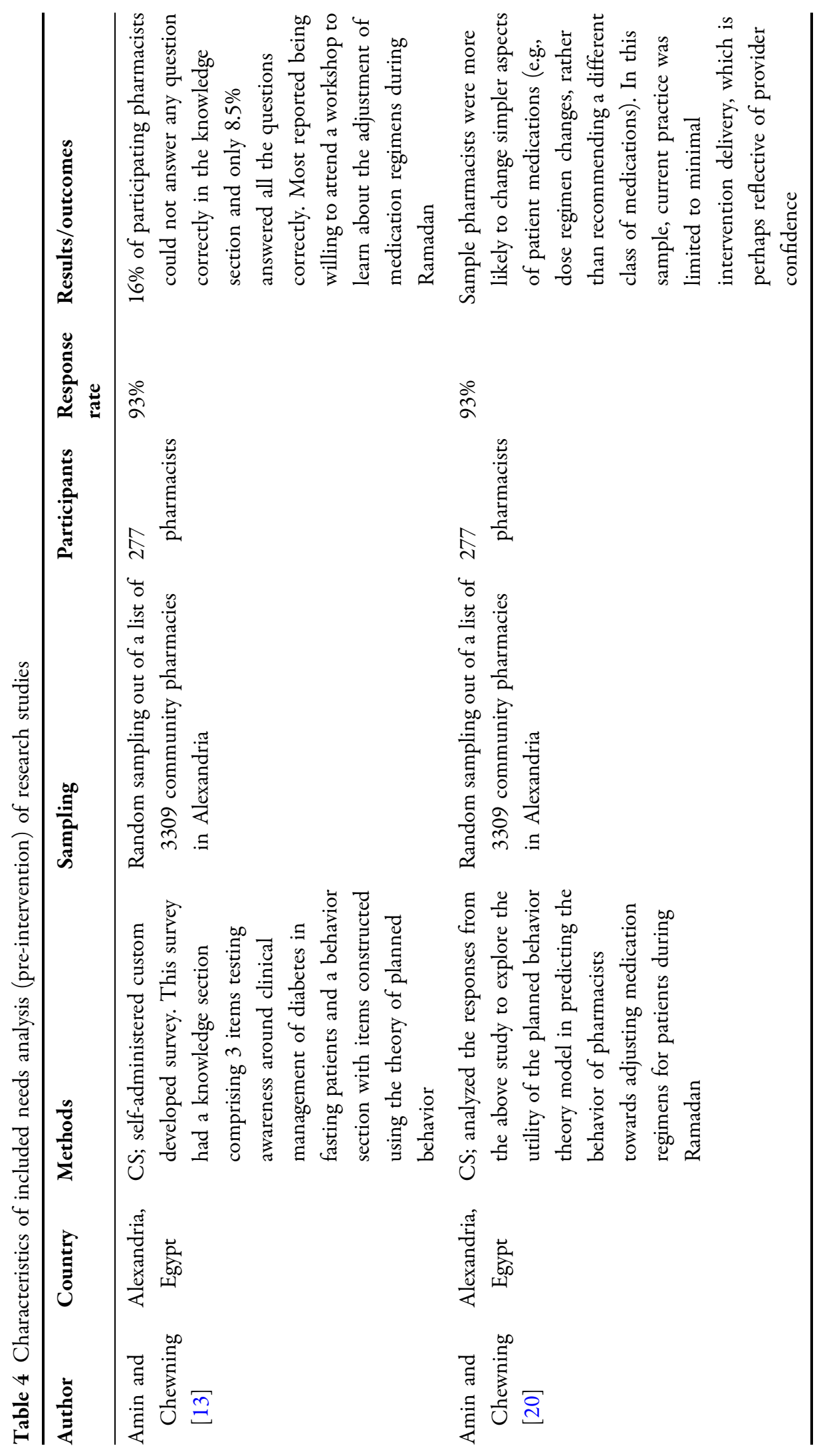




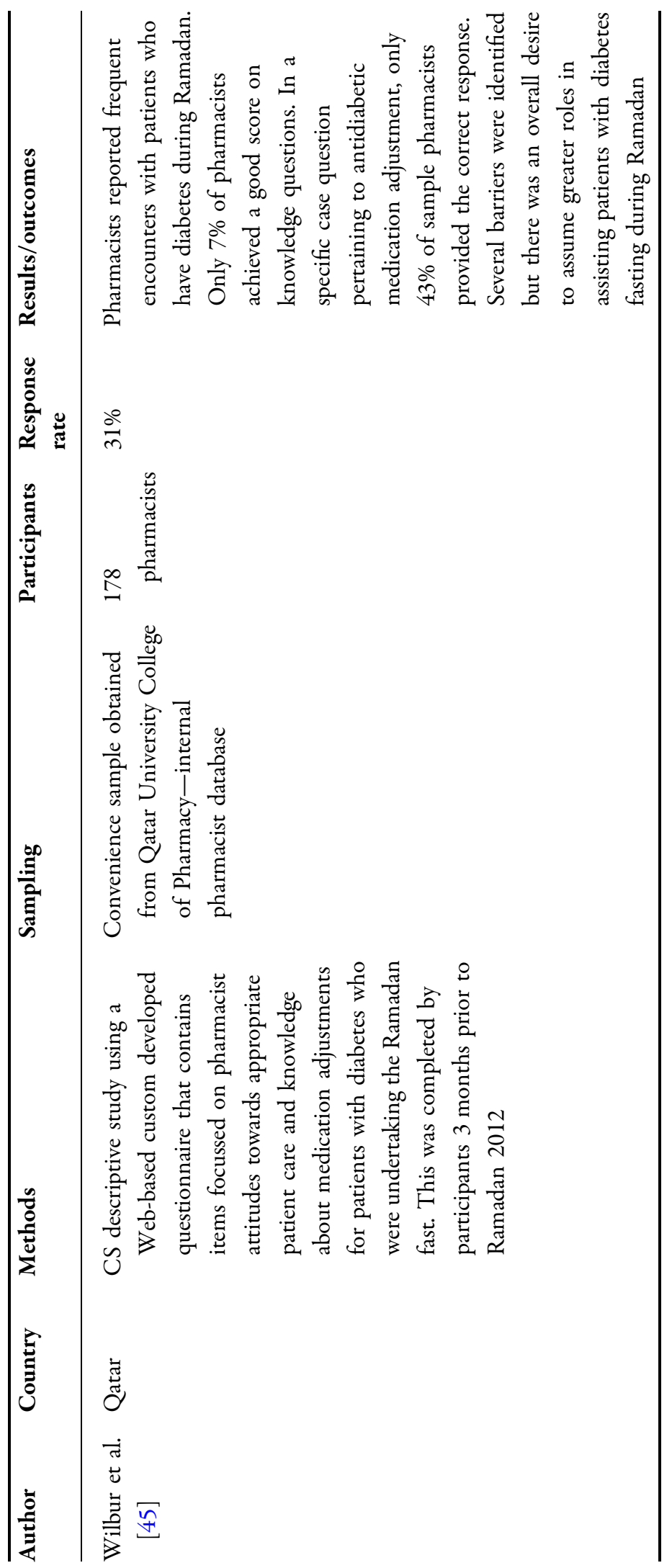




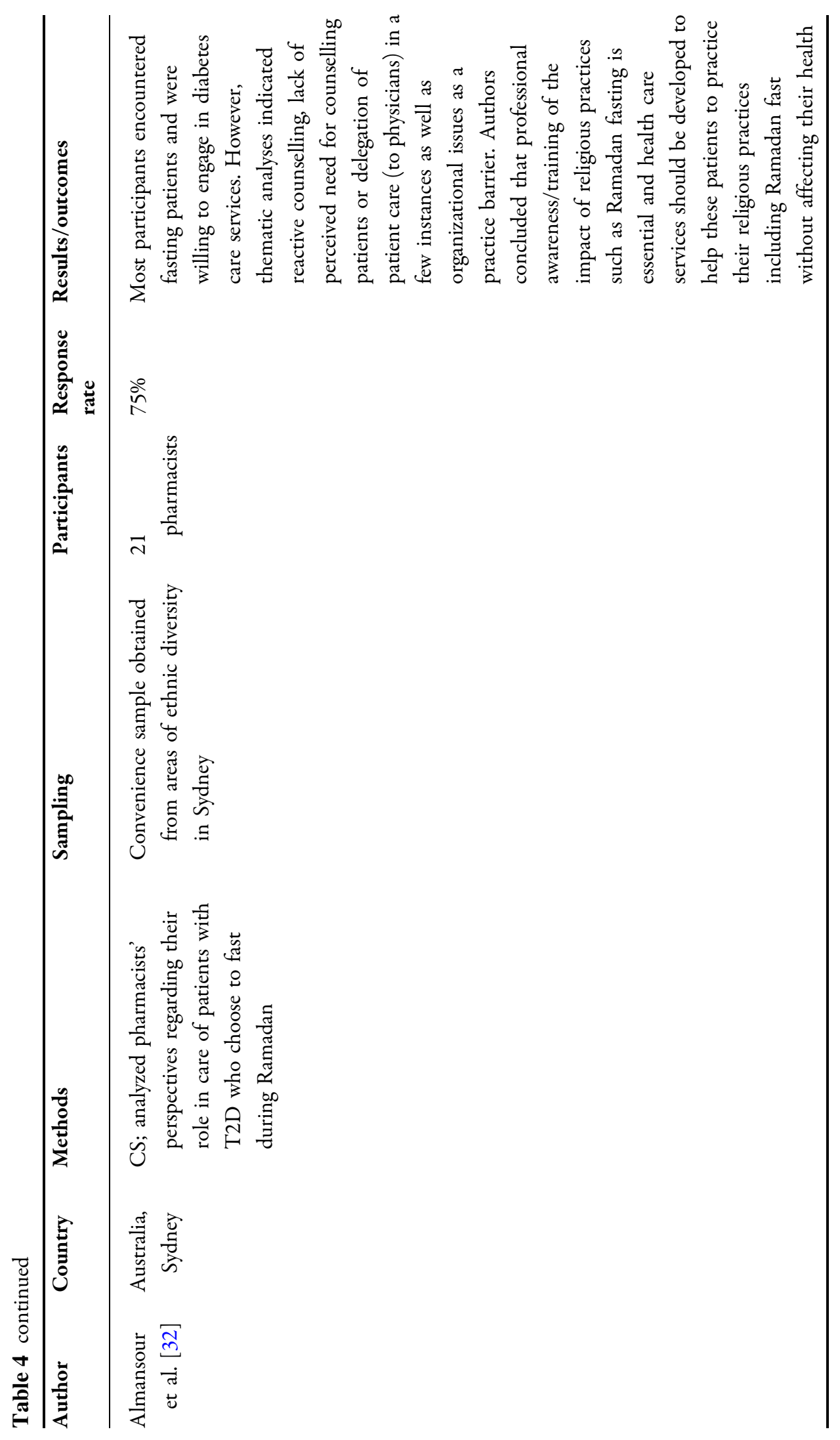




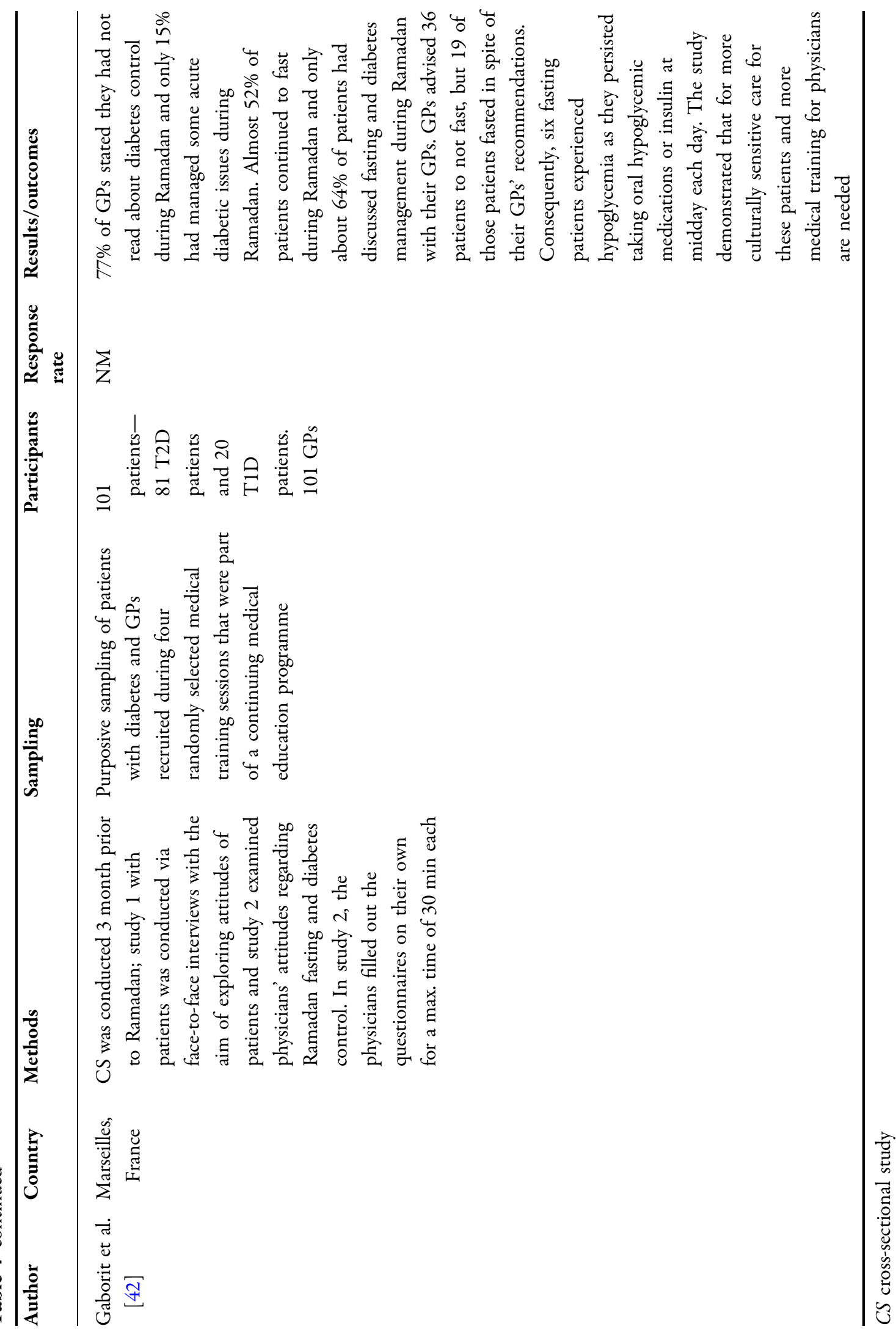


many instances. None of the included interventions were large robust randomized control trials (RCTs), some were pre-post studies and others were parallel group quasi-experimental studies [23, 25, 34-40].

\section{Intervention Specifics}

Most interventions targeted patients with diabetes who intended to or have the intention to fast during Ramadan. Interventions were mostly delivered by HCPs such as GPs, dieticians, and link workers and a few involved spiritual leaders to recruit or motivate patients to participate in such interventions. Most intervention studies stated focussing on patients' education on meal planning, physical activity, glucose monitoring, hypoglycemia, dosage, and the timing of medications. However, in most instances, the details of the educational intervention, e.g., the format of delivery, props used to support the education, or the clinical guidelines which informed the educational interventions, were not detailed. A few studies reported the language and the duration of the education sessions [34, 36, 39]. In multicenter studies, no quality audits were considered [36, 40]. Hence in these instances protocol fidelity is not known.

\section{Key Outcomes Measured}

Intervention assessment is best served by measuring a balanced set of outcomes that include clinical measures, economic benefits as well as functional health status and well-being [41]. Key clinical outcomes measured in the reviewed studies included weight or BMI, HbA1c, and adverse events occurrence. However, humanistic outcomes (e.g., adherence, quality of life, satisfaction, health beliefs, awareness, behaviors, and attitudes) were not considered to assess the effects of the interventions. Therefore, these studies did not look into details of whether participating patients learnt/benefited from these studies outside the bounds of clinical improvement. In most instances, the follow-up period was short and only one study looked into the sustainability of the outcomes 12 months after patients attended the education [39]. In this study, i.e., the Ramadan Education and Awareness in Diabetes (READ) program, HbA1c reduction was sustained in the intervention group $(-0.13 \%, p=0.07)$, while the mean HbA1c in the control group increased by $0.33 \%$ $(p=0.03)$ [39].

Effect sizes of the educational interventions on key outcomes, where calculable, ranged between -1.14 and 1.7.

\section{Needs Analysis (Pre-intervention) Studies}

Needs analysis research studies were conducted both with GPs and pharmacists, and patients. None of these included active intervention studies. Non-validated instruments, self-reporting methods, sampling in one area, and limited scope of questions on diabetes knowledge were issues that restricted the generalizability of the results. While most HCPs were willing to provide health care services, clearly their knowledge of how such services will be provided was suboptimal, and most were open to more training about their role in managing diabetes during Ramadan. There was a wide variation in the knowledge of participating HCPs (GPs and pharmacists) about managing diabetes for patients with diabetes intending to fast during Ramadan. For example, the majority of the French GPs interviewed by Gaborit et al. lacked the clinical awareness about medical management in T2D patients observing Ramadan and cultural awareness about the relevance of the fast [42].

\section{DISCUSSION}

This is the first literature review to focus specifically on the health educational interventions, and intentions of HCPs such as general practitioners (GPs) and pharmacists, to provide clinical services to patients with diabetes while fasting during Ramadan. The review revealed a paucity of research in this area. There were only a few intervention studies located through the search, and the generalizability of these studies was limited. Power calculations were not undertaken for most intervention studies, making it difficult to extrapolate the 
significance of findings. Outcome measures collected varied and humanistic outcomes were often not collected. A detailed description of actual intervention or education was lacking, and protocol fidelity (e.g., checks to see if intervention providers followed research protocols) and assessment of patient adherence (checks to see if patients adhered to recommended interventions) to recommendations were not measured in any of the reviewed studies. The overall trend, however, indicated that education, counselling by HCPs, and dosage adjustment for oral hypoglycemics in the pre-Ramadan period help decrease adverse events and can thus improve the experience of patients choosing to fast for religious/spiritual reasons. Clearly, robust studies with attention to trial design need to be conducted.

None of the included studies had a robust design, some were pre-post studies and others were parallel group quasi-experimental studies $[23,25,34-40]$. In studies where randomized sampling was undertaken, the randomization method, allocation concealment, or blinded outcome collection are not mentioned. In terms of outcomes, the full gamut of clinical and humanistic outcomes was not covered and certainly economic analyses, e.g., the cost-effectiveness of the educational interventions, were also not conducted. One reason for this of course may have been the lack of validated questionnaires/tools to measure humanistic outcomes such as participants' knowledge, satisfaction, and quality of life with these interventions. These studies used self-developed questionnaires. Future intervention studies in this area should consider adequately powered randomized controlled designs, with blinding of outcome measurements at least as well as a suite of outcomes (clinical, humanistic, and economic) measured using validated instruments or techniques.

In most of the intervention studies, HbA1c or blood glucose level and diabetes-related adverse events were improved in the intervention group. These are promising outcomes, even though study designs were not very robust, as mentioned earlier. The positive trends in the data observed suggested that well-designed health care education models for Ramadan observers with diabetes should be developed. However, a preceding step that may need research investment is the development and validation of measurement tools that can be used in this population, particularly for measuring humanistic outcomes.

As highlighted in the "Results" section, intervention/education details were not clearly described, which prevents other researchers from using effective materials and having to reinvent the intervention. These details include the period of how long the education took, method of delivery (e.g., power point slides, face to face), and whether clinical guidelines were followed in constructing the educational intervention. Key clinical details of interventions were missing. For example, smoking affects enzymes inducers, and smokers who fast during Ramadan may stop smoking suddenly during the fasting time, which might affect the doses of some medications. However, none of the intervention studies discussed whether dose adjustment in relation to smoking cessation had been advised to patients. This is an issue observed by authors of other systematic reviews, e.g., Okumura et al. reported the same issue in a review of intervention trials focusing on counselling [43]. Future research needs to consider these details to enable other researchers to implement such research in other countries for more global benefits and universal standardization.

Most of the interventions were delivered by physicians and allied health professionals in clinics. In most communities around the world, pharmacists are knowledgeable HCPs, very easily accessible and commonly visited by many people every day. Therefore, pharmacists' valuable contribution to patient care can be better utilized. Research has shown that community pharmacists can help with diabetes management generally $[29,44]$. Therefore, it is logical that community pharmacists can help people with diabetes who are observing the fast of Ramadan by providing information and helping to adjust medications. Pharmacists' awareness of and willingness to do so was evident to some extent in the needs analysis studies included in this review [13, 20, 32, 42]. There were no studies describing the effect of well-designed 
structured clinical interventions delivered by pharmacists to patients with diabetes fasting during Ramadan.

The studies that were located in the literature had several limitations; however, put together, these needs analysis (pre-intervention), in the case of those conducted with pharmacists', highlighted that pharmacists have the ability to enact specific roles in adjustments of medication regimens for patients observing fasts with concomitant diabetes [13, 20, 32, 45]. Hence, it would be ideal to upskill pharmacists so they can adjust medication regimens for patients to ensure that they take their medications safely and appropriately before, during, and after Ramadan.

Patient education research often highlights the chasm between patient behaviors and professionals' recommendations. Several cultural factors may affect communication and counselling by health professionals and similar factors may also influence patients' knowledge about their condition/medications as well as their help-seeking behaviors. Thus, several types of interventions may be useful: enhancing cultural understanding of professionals about the importance of the fast to patients in developed countries, as well as clinical training about adjustment of medication regimens for patients with diabetes opting to fast during Ramadan in both developed and developing countries. Ultimately, patients themselves can be upskilled to self-manage medications through effective patient education and medication adjustment skills. Very few studies in this review directly addressed both provider and patient attitudes. The work reported by Gaborit and colleagues [42] was the only study in the review which evaluated attitudes of both patients and physicians regarding Ramadan fasting and diabetes control in Marseilles, France. As highlighted in Table 3, the authors reported that many GPs lacked the cultural competency and medical knowledge that are needed to appropriately counsel their patients with diabetes in regards to medication management during Ramadan. This resulted in medically unjustified recommendations against fasting. Culturally insensitive recommendations were ineffective, in that, directing patients peremptorily not to fast did not appear to deflect vulnerable patients from still undertaking the fast [42]. Generally, patients may choose to ignore HCP advice, especially if they feel the HCP is not culturally attuned to their decisions [42, 46, 47]. In the case of Ramadan fasting, where religious beliefs strongly motivate patients to fast, HCPs (physicians and pharmacists) and religious sources, such as imams (Muslim religious priests), may need to collaborate as important sources of knowledge about fasting and medication use for patients. This is a research area with clear gaps, and further research on both patient-focussed or health professional-focussed research is needed.

A few studies have been carried out in Australia regarding diabetes management in CALD populations (i.e., Maltese immigrants to Australia), but not specifically during fasting. One of these studies reported on interview data from Maltese immigrants in Sydney, Australia. This was a qualitative exploratory study and highlighted how patients in this group have limited diabetes knowledge. Interestingly, this group of patients were interested to receive more diabetes care and counselling from community pharmacists as they are easily accessible [44]. It was concluded that CALD populations have distinctive barriers to health care that might be unknown to HCPs or unrecognized clearly by patients themselves. Some of these barriers could be due to low health literacy or lack of access to educational programs [44]. The effect of a community pharmacy diabetes service model on the outcomes of general patients with T2D in Australia has been assessed. As a result of these significant outcomes, the Australian government provided a clinical intervention fee for pharmacists to provide an abbreviated version of this service in the Fifth Community Pharmacy Agreement [48]. Such programs could also be developed for niche population groups as well, i.e., specifically for Muslim patients with diabetes intending to fast in Ramadan. Also, future research may consider electronic online formats of resources for patient education and awareness such as smartphone apps (for iOS, iPhone, and Android) as digital tools are used commonly worldwide. Contextualized exploratory research seeking patients' perspectives of 
problems or cultural issues regarding diabetes self-control would need to be conducted prior to designing such pharmacy-based intervention programs. Pharmacists' perspectives about dealing with cultural concerns that could affect patients' health or clinical confidence in providing specific interventions was investigated in Australia for this group and the findings of such work could be beneficial in the planning phase of such intervention programs [32].

There are a few limitations of this scoping review. Meta-analysis could not be conducted because the reviewed studies had mixed methods/protocols of the interventions. Similarly, a systematic review was not conducted as most of the included studies were not robustly designed clinical trials. Another limitation is that diabetes was considered mostly to be studied with relation to Ramadan fast, because of the possibility of hypoglycemia occurring while fasting. However, other health conditions such as angina, ulcers, asthma, and chronic obstructive pulmonary disease (COPD) should be considered in future studies as fasting may affect health and medication use in relation to other conditions.

\section{CONCLUSION}

The results of this literature review revealed numerous gaps in the existing literature. Many HCPs and patients lack the knowledge for the management and modification of medication regimens for patients with diabetes who fast during Ramadan. Addressing the knowledge deficits and enhancing cultural competency are critical for clinicians treating Muslim patients living with chronic illness and observing the fasting practices of their faith. Very few studies, however, have addressed health providers' attitudes, knowledge, and advice with regards to fasting. In Australia, where thousands of Muslims observe the fast of Ramadan each year, this research area is becoming increasingly important.

Pharmacists have been the focus of research in this area in other countries and have been shown to be willing to provide specialized care for fasting patients with diabetes and willing to upskill themselves in order to provide such specialized care. Hence, there is a need for research that helps to describe the extent to which Australian pharmacists counsel their patients about fasting during Ramadan, their willingness to provide specialized care, and their level of current cultural and clinical skills. Similarly, research with Muslim Australian patients observing Ramadan will help establish their Ramadan-based diabetes self-management behaviors, and their perceptions about, and willingness for receiving specialized help from clinicians, including pharmacists. This research might assist in the development of such programs in Australia.

\section{ACKNOWLEDGEMENTS}

No funding or sponsorship was received for this study or publication of this article. All named authors meet the International Committee of Medical Journal Editors (ICMJE) criteria for authorship for this manuscript, take responsibility for the integrity of the work as a whole, and have given final approval for the version to be published.

Disclosures. H. A. Almansour, B. Chaar, and B. Saini have nothing to disclose.

Compliance with Ethics Guidelines. This article is based on published studies, and does not involve any new studies of human or animal subjects performed by any of the authors.

Open Access. This article is distributed under the terms of the Creative Commons Attribution-NonCommercial 4.0 International License (http://creativecommons.org/licenses/ by-nc/4.0/), which permits any noncommercial use, distribution, and reproduction in any medium, provided you give appropriate credit to the original author(s) and the source, provide a link to the Creative Commons license, and indicate if changes were made. 


\section{REFERENCES}

1. Lee SW, Lee JY, Tan CS, Wong CP. Strategies to make Ramadan fasting safer in type 2 diabetics: a systematic review and network meta-analysis of randomized controlled trials and observational studies. Med (Baltimore). 2016;95(2):e2457.

2. Ali S, Davies MJ, Brady EM, et al. Guidelines for managing diabetes in Ramadan. Diabet Med. 2016;33(10):1315-29.

3. Australian Institute of Health and Welfare (AIHW). How common is diabetes? 2013. http://www.aihw. gov.au/how-common-is-diabetes/. Accessed 3 May 2015.

4. University of South Australia. Australian Muslims: a demographic, social and economic profile of Muslims in Australia. 2015. https://www.unisa. edu.au/Global/EASS/MnM/Publications/Australian_ Muslims_Report_2015.pdf. Accessed 15 Aug 2016.

5. Australian Bureau of Statistics (ABS). Reflecting a nation: stories from the 2011 census, 2012-2013. http://www.abs.gov.au/ausstats/abs@.nsf/Lookup/ 2071.0main+features902012-2013. Accessed 2 May 2015.

6. Thow AM, Waters AM. Diabetes in culturally and linguistically diverse Australians: identification of communities at high risk. 2005. http://www.aihw. gov.au/WorkArea/DownloadAsset.aspx?id=6442454 961. Accessed 5 May 2015.

7. Trepanowski JF, Bloomer RJ. The impact of religious fasting on human health. Nutr J. 2010;9:57.

8. Aadil N, Houti IE, Moussamih S. Drug intake during Ramadan. BMJ. 2004;329(7469):778-82.

9. Al-Arouj M, Assaad-Khalil S, Buse J, et al. Recommendations for management of diabetes during Ramadan: update 2010. Diabetes Care. 2010;33(8):1895-902.

10. Hui E, Bravis V, Hassanein M, et al. Management of people with diabetes wanting to fast during Ramadan. BMJ. 2010;340:c3053.

11. Pathy R, Mills KE, Gazeley S, Ridgley A, Kiran T. Health is a spiritual thing: perspectives of health care professionals and female Somali and Bangladeshi women on the health impacts of fasting during Ramadan. Ethn Health. 2011;16(1):43-56.

12. Salti I, Bénard E, Detournay B, et al. A population-based study of diabetes and its characteristics during the fasting month of Ramadan in 13 countries. Diabetes Care. 2004;27(10):2306-11.
13. Amin ME, Chewning B. Community pharmacists' knowledge of diabetes management during Ramadan in Egypt. Int J Clin Pharm. 2014;36(6):1213-21.

14. Alberti H, Boudriga N, Nabli M. Lower attendance rates and higher fasting glucose levels in the month of Ramadan in patients with diabetes in a Muslim country. Diabet Med. 2008;25(5):637-8.

15. Al-Mendalawi MD. Effects of Ramadan fasting on glucose levels and serum lipid profile among type 2 diabetic patients. Saudi Med J. 2011;32(6):647.

16. Chan F, Slater C, Syed AA. Diabetes and Ramadan. Fasts after bariatric surgery. BMJ. 2010;341: c3706.

17. Khaled BM, Belbraouet S. Effect of Ramadan fasting on anthropometric parameters and food consumption in 276 type 2 diabetic obese women. Int J Diabetes Dev Ctries. 2009;29(2):62-8.

18. Salman H, Abdallah MA, Abanamy MA, Howasi M. Ramadan fasting in diabetic children in Riyadh. Diabet Med. 1992;9(6):583-4.

19. Karatoprak C, Yolbas S, Cakirca M, et al. The effects of long term fasting in Ramadan on glucose regulation in type 2 diabetes mellitus. Eur Rev Med Pharmacol Sci. 2013;17(18):2512-6.

20. Amin ME, Chewning B. Predicting pharmacists' adjustment of medication regimens in Ramadan using the theory of planned behavior. Res Social Adm Pharm. 2015;11(1):e1-15.

21. Norouzy A, Mohajeri SM, Shakeri S, et al. Effect of Ramadan fasting on glycemic control in patients with type 2 diabetes. $\mathrm{J}$ Endocrinol Invest. 2012;35(8):766-71.

22. Alabbood MH, Ho KW, Simons MR. The effect of Ramadan fasting on glycaemic control in insulin dependent diabetic patients: a literature review. Diabetes Metab Syndr. 2016. doi:10.1016/j.dsx. 2016.06.028

23. Ahmedani MY, Haque MS, Basit A, Fawwad A, Alvi SF. Ramadan prospective diabetes study: the role of drug dosage and timing alteration, active glucose monitoring and patient education. Diabet Med. 2012;29(6):709-15.

24. Ibrahim M, Abu Al Magd M, Annabi FA, et al. Recommendations for management of diabetes during Ramadan: update 2015. BMJ Open Diabet Res Care. 2015;3(1):e000108.

25. Ahmedani MY, Alvi SF, Haque MS, Fawwad A, Basit A. Implementation of Ramadan-specific diabetes management recommendations: a multi-centered 
prospective study from Pakistan. J Diabetes Metab Disord. 2014;13(1):37.

26. Ali M, Schifano F, Robinson $\mathrm{P}$, et al. Impact of community pharmacy diabetes monitoring and education programme on diabetes management: a randomized controlled study. Diabet Med. 2012;29(9):e326-33.

27. Jacobs M, Sherry PS, Taylor LM, Amato M, Tataronis GR, Cushing G. Pharmacist assisted medication program enhancing the regulation of diabetes (PAMPERED) study. J Am Pharm Assoc. 2012;52(5):613-21.

28. Krass I, Armour CL, Mitchell B, et al. The pharmacy diabetes care program: assessment of a community pharmacy diabetes service model in Australia. Diabet Med. 2007;24(6):677-83.

29. Mitchell B, Armour C, Lee M, et al. Diabetes medication assistance service: the pharmacist's role in supporting patient self-management of type 2 diabetes (T2DM) in Australia. Patient Educ Couns. 2011;83(3):288-94.

30. Wang Y, Yeo QQ, Ko Y. Economic evaluations of pharmacist-managed services in people with diabetes mellitus: a systematic review. Diabet Med. 2016;33(4):421-7.

31. Watson LL, Bluml BM. Integrating pharmacists into diverse diabetes care teams: implementation tactics from project IMPACT. Diabetes J Am Pharm Assoc. 2014;54(5):538-41.

32. Almansour HA, Chaar B, Saini B. Pharmacists' perspectives about their role in care of patients with diabetes observing Ramadan. Res Social Adm Pharm. 2017;13(1):109-22.

33. Peters MD, Godfrey CM, Khalil H, McInerney P, Parker D, Soares CB. Guidance for conducting systematic scoping reviews. Int J Evid Based Healthc. 2015;13(3):141-6.

34. Susilparat P, Pattaraarchachai J, Songchitsomboon S, Ongroongruang S. Effectiveness of contextual education for self-management in Thai Muslims with type 2 diabetes mellitus during Ramadan. J Med Assoc Thai. 2014;97(Suppl 8):S41-9.

35. Siaw MY, Chew DE, Dalan R, et al. Evaluating the effect of Ramadan fasting on muslim patients with diabetes in relation to use of medication and lifestyle patterns: a prospective study. Int J Endocrinol. 2014;2014:308546.

36. McEwen LN, Ibrahim M, Ali NM, et al. Impact of an individualized type 2 diabetes education program on clinical outcomes during Ramadan. BMJ Open Diabet Res Care. 2015;3(1):e000111.
37. Lee JY, Lee SW, Nasir NH, How S, Tan CS, Wong CP. Diabetes telemonitoring reduces the risk of hypoglycaemia during Ramadan: a pilot randomized controlled study. Diabet Med. 2015;32(12):1658-61.

38. Fatim J, Karoli R, Chandra A, Naqvi N. Attitudinal determinants of fasting in type 2 diabetes mellitus patients during Ramadan. J Assoc Phys India. 2011;59:630-4.

39. Bravis V, Hui E, Salih S, Mehar S, Hassanein M, Devendra D. Ramadan Education and Awareness in Diabetes (READ) programme for Muslims with type 2 diabetes who fast during Ramadan. Diabet Med. 2010;27(3):327-31.

40. Ahmedani MY, Alvi SF. Characteristics and Ramadan-specific diabetes education trends of patients with diabetes (CARE): a multinational survey (2014). Int J Clin Pract. 2016;70(8):668-75.

41. Kozma CM, Reeder CE, Schulz RM. Economic, clinical, and humanistic outcomes: a planning model for pharmacoeconomic research. Clin Ther. 1993;15(6):1121-32.

42. Gaborit B, Dutour O, Ronsin O, et al. Ramadan fasting with diabetes: an interview study of inpatients' and general practitioners' attitudes in the South of France. Diabetes Metab. 2011;37(5):395-402.

43. Okumura LM, Rotta I, Correr CJ. Assessment of pharmacist-led patient counseling in randomized controlled trials: a systematic review. Int J Clin Pharm. 2014;36(5):882-91.

44. Barbara S, Krass I. Self management of type 2 diabetes by Maltese immigrants in Australia: can community pharmacies play a supporting role? Int J Pharm Pract. 2013;21(5):305-13.

45. Wilbur K, Al Tawengi K, Remoden E. Diabetes patient management by pharmacists during Ramadan. BMC Health Serv Res. 2014;14:117.

46. Mir G, Sheikh A. 'Fasting and prayer don't concern the doctors-they don't even know what it is': communication, decision-making and perceived social relations of Pakistani Muslim patients with long-term illnesses. Ethn Health. 2010;15(4):327-42.

47. Mygind A, Kristiansen M, Wittrup I, Norgaard LS. Patient perspectives on type 2 diabetes and medicine use during Ramadan among Pakistanis in Denmark. Int J Clin Pharm. 2013;35(2):281-8.

48. The Pharmacy Guild of Australia. Fifth Community Pharmacy Agreement (2010-2015). 2010. http://guild. 
org.au/the-guild/community-pharmacy-agreement. Accessed 15 May 2015.

49. Karamat MA, Syed A, Hanif W. Review of diabetes management and guidelines during Ramadan. J R Soc Med. 2010;103(4):139-47.
50. Chinn S. A simple method for converting an odds ratio to effect size for use in meta-analysis. Stat Med. 2000;19(22):3127-31. 